\title{
Dynamiques électorales et forces politiques en Toscane
}

Mr Mario Caciagli, Mr Carlo Baccetti, Mr Emmanuel Négrier

\section{Citer ce document / Cite this document :}

Caciagli Mario, Baccetti Carlo, Négrier Emmanuel. Dynamiques électorales et forces politiques en Toscane. In: Pôle Sud, $n^{\circ} 8$, 1998. Elections et politiques régionales. pp. 86-97;

doi : https://doi.org/10.3406/pole.1998.996

https://www.persee.fr/doc/pole_1262-1676_1998_num_8_1_996

Fichier pdf généré le 23/04/2018 


\section{Dynamiques électorales et forces politiques en Toscane}

par Mario Caciagli et Carlo Baccetti

Université de Florence (traduit de l'italien par E. Négrier)

Pôle Sud N 8-Mai $1998-p .86$ à 97.

Cet article fait le bilan de dix années de vie politique en Toscane. Dans une région traditionnellement marquée par une orientation à gauche, les effets des renouvellements partisans et du passage à la IF République ont été sensiblement moins forts qu'ailleurs. Ceci tient largement dans les modes de relation entre societté civile, territoire et système politique. Même si l'on constate le succès de la recomposition autour du centre-gauche et la faiblesse générale des forces situées à droite de l'échiquier politique, il est incontestable que l'ancrage du PDS, et même de Rifondazione Comunista, dans la société toscane n'a que peu à voir avec celui qui, autour de l'hégémonie du PCI, faisait de la Toscane une "Région rouge " par excellence.

\section{Une région rouge}

On présente souvent la Toscane comme une des régions rouges italiennes, où l'ampleur et la continuité d'une tendance favorable à la gauche s'expliqueraient par la fidélité à une tradition remontant au fascisme, et par la présence d'une solide structure de grands partis de masse soutenus par une multitude d'organismes parallèles (Caciagli, 1995). La culture politique socialement dominante a fourni à la région une forte identité collective et un degré élevé de politisation, illustrés par des taux records de syndicalisation et de militantisme politique, un réseau d'associations politiques et un taux de participation électorale de première importance.

Après-guerre, le $\mathrm{PCI}$ avait conquis une position dominante au sein de la gauche et du spectre politique régional. Au long des décennies, il avait recueilli entre $40 \%$ et $45 \%$ des suffrages, pour 200000 membres environ, soit $15 \%$ du total italien. Le PSI, puissant dans l'immédiat après-guerre, subit, au cours des décennies, une chute d'influence, tout en atteignant environ 12$13 \%$ des voix, pour un nombre de militants plus modeste : 40000 adhérents en moyenne, soit 6 à $8 \%$ du total italien. Ladversaire du PCI, la DC, n'est jamais parvenue à se hisser au-delà de sa condition minoritaire. Au contraire, son influence déclina jusqu'à atteindre seulement $25 \%$ dans les années 1980. Ses adhérents, 50000 en moyenne, ne représentaient que $3 \%$ à peine de la moyenne nationale, et étaient en grande partie originaires de la province de Lucca, la seule zone régionale où, grâce à une subculture catholique, le parti de gouvernement italien était parvenu à maintenir pendant 40 ans les conditions de sa domination. Le MSI, néo-fasciste, a toujours été marginalisé, avec une influence électorale proche de sa moyenne nationale (4-5\%). Pendant les diverses phases de son histoire récente, on note la présence, modeste mais significative, de groupes dissidents du PSI, 


\section{Dynamiques électorales et forces politiques en Toscane}

socio-démocrates ou socialistes de gauche. Rappelons que, fidèles aux traditions läques toscanes, quelques zones côtières connaissaient une vieille tradition de vote républicain (PRI), en déclin cependant au cours des dernières années.

Pendant des décennies, le PCI et le PSI ont gouverné la grande partie des communes et des provinces, et, dès sa constitution en 1970, la Toscane. À plusieurs reprises, le PCI a dû, selon une logique de coalition, concéder au PSI des mairies de villes importantes, et la présidence de la région elle-même. Seule Florence a toujours été gouvernée par des coalitions excluant le PCI, alors que Lucca et presque toutes les communes de cette province étaient dirigées par les démocrateschrétiens.

Les fondements de la domination du PCI remontent à l'immédiat après-guerre, à la suite de la Résistance et grâce au soutien du groupe social majoritaire en Toscane : les métayers. La transformation de la Toscane d'une région agricole à une région industrielle, à partir de la fin des années 1950, et les profondes transformations sociales qui en

\section{Toscane et Italie.}

Résultats des référendums de 1985 et 1990 (en \%)

1985 (INDEXATION DU COUT DE LA VIE)

$\begin{array}{lcc} & \text { Toscane } & \text { Italie } \\ \text { Oui (pour l'abrogation) } & 55,2 & 45,7 \\ \text { Non (contre l'abrogation) } & 44,8 & 54,3 \\ \text { Votants } & 86,9 & 77,9 \\ \text { Nuls } & 2,4 & 3,2\end{array}$

\begin{tabular}{|c|c|c|}
\hline \multicolumn{3}{|c|}{1990 (REGLEMENTATION DE LA CHASSE) } \\
\hline & Toscane & Italie \\
\hline Oui (pour l'abrogation) & 91,2 & 92,2 \\
\hline Non (contre l'abrogation) & 8,8 & 7,8 \\
\hline Votants & 33,5 & 43,4 \\
\hline Nuls & 5,3 & 5,8 \\
\hline
\end{tabular}

ont découlé, ont renforcé le PCI, qui s'est montré habile à conjuguer tradition et capacité de gouvernement.

Alors que son déclin électoral était déjà entamé en Italie, le PCI a obtenu en Toscane son dernier grand succès lors du référendum sur l'échelle mobile en 1985 (tableau $n^{\circ} 1$ ).

Dans les années suivantes, les résultats d'élections partielles et, surtout, des législatives de 1987, signalent pourtant le début d'une crise qui aboutira plus tard au tournant de 1989 et au démantèlement du parti lui-même.

Si la DC ni le PSI, eux-mêmes en crise au niveau national, n'ont su profiter de cette crise du PCI, la naissance du PDS et de Rifondazione Comunista paraissaient sanctionner le déclin de la subculture rouge. Les événements ultérieurs, avec la fin de la Première République, les nouveaux systèmes électoraux et la naissance du bloc de centredroit ont au contraire favorisé en Toscane le regain de la gauche. La coalition de l'Olivier a ainsi permis à celle-ci de s'ouvrir au centre laïc et catholique et d'étendre une influence telle que cette région conserve une physionomie de gauche.

\section{Le grand tournant de 1989-1991}

Après le grand succès de 1975-1976, la stagnation des scores du PCI entre 1979 et 1985 semblait normale pour la Toscane, d'autant plus si on la comparait à son effondrement au sud et dans les grandes villes du nord. Son triomphe lors des élections européennes de 1984, puis du référendum de 1985 , a trompé les communistes quant au maintien de leur influence. La chute de $3 \%$ en 1987 par rapport à 1983 fut aussi grave que surprenante, mais c'était surtout la 


\section{Pôle Sud $N^{\circ} 8$}

première en quarante ans. La suite révéla que c'étaient en fait les premiers signes d'un tournant que rien ne laissait pourtant prévoir, entre 1989 et 1991.

En novembre 1989 , la proposition d'Achille Occhetto de changer le nom du parti provoqua un grand trouble chez les militants, les inscrits et les électeurs toscans. Dans la perspective du congrès extraordinaire de mars 1990 , la Toscane, avec son nombre d'inscrits élevé, contribua largement à la ratification de la proposition d'Occhetto, mais les oui en faveur du projet du secrétaire général ne furent nombreux que dans les zones les plus rouges où la participation aux comités du Congrès fut plus faible. Là où la présence des inscrits fut la plus élevée, cela signifiait aussi l'existence des fractures les plus profondes, des débats plus soutenus et des conflits ouverts dans les comités de base du Congrès. Dans tout le parti régional, la division fut profonde et lancinante (Baccetti, 1991).

Le malaise ressenti par la base se révéla dans toute son étendue à l'occasion des élections locales et régionales de mai 1990 , lorsque le PCI perdit plus de 6 points par rapport aux précédentes (1985); une baisse exceptionnelle sous la Première République. L'augmentation des abstentions, des votes nuls et blancs a été attribuée au trouble de l'électorat communiste, dont les autres partis n'ont su profiter (tableau 2).

Dans cette période, la culture politique rouge semble victime d'un profond traumatisme : même dans les communes les plus rouges de la province, la participation électorale, haute dans le passé, cesse d'être une vertu. La manifestation la plus claire de cela se situe au mois de juin, à l'occasion du réfé- rendum abrogatif d'une loi limitant l'exercice de la chasse, pour lequel la passion de nombre de militants et d'électeurs communistes n'avait d'égal que les hésitations du parti sur la question : le résultat fut un score élevé d'abstentions, qui fit tomber à 33,5\% la participation (Tableau 1) d'un électorat réputé avoir un comportement inverse (Baccetti, Caciagli, 1994).

En 1991, la dissolution du PCI et la disparition de l'URSS ont paru porter le coup mortel à une longue tradition agrégeant à la fois la totalité du cadre politique et les dynamiques électorales de la région. Cette annéelà, il semblait que la crise de la culture rouge fût définitive. Le parti, point de référence

\begin{tabular}{|c|c|c|c|}
\hline $\begin{array}{l}\text { 2. Toscane. E } \\
1985-199\end{array}$ & ection & $\begin{array}{l}\text { gional } \\
n \%)\end{array}$ & \\
\hline PARTIS & 1985 & 1990 & 1995 \\
\hline PDS & & & 40,9 \\
\hline PRC & & & 11,1 \\
\hline PCI & 46,2 & 39,8 & \\
\hline $\mathrm{DP}$ & 1,4 & 1,1 & \\
\hline Verdi & 1,6 & 3,8 & 2,7 \\
\hline Federazione Laburista & & & 1,4 \\
\hline PSI & 12,0 & 13,6 & \\
\hline PSDI & 1,7 & 1,6 & \\
\hline PRI & 3,3 & 3,5 & 0,8 \\
\hline $\mathrm{DC}$ & 26,6 & 25,9 & \\
\hline Popolari-Patto democratico & & & 6,4 \\
\hline $\mathrm{CCD}$ & & & 2,4 \\
\hline Lista Antiproibizionista & & 1,0 & \\
\hline Lista Panella-Riformatori & & & 1,3 \\
\hline PLI & 1,1 & 1,0 & \\
\hline Forza Italia-Polo popolare & & & 19,1 \\
\hline MSI-DN & 4,6 & 3,3 & \\
\hline $\mathrm{AN}$ & & & 13,1 \\
\hline Lega Nord* & 0,5 & 0,8 & 0,7 \\
\hline CPA & & 3,1 & \\
\hline Autres & 1,0 & 1,4 & \\
\hline Votants & 92,8 & 89,6 & 85,2 \\
\hline Nuls & 4,9 & 6,9 & 17,2 \\
\hline Dont blancs & 2,7 & 3,5 & 8,6 \\
\hline
\end{tabular}

* En 1985 Liga Veneta-Alleanza Italiana pensionati; en 1990 Lega lombarda. 


\section{Dynamiques électorales et forces politiques en Toscane}

pour des générations de Toscans, n'existait plus; les syndicats et les pouvoirs locaux ont alors été accusés de n'être plus capables d'affronter les nouveaux défis et de résoudre les problèmes locaux les plus cruciaux; les Maisons du Peuple et les fêtes de l'Unità semblaient avoir perdu beaucoup de leurs fonctions de communication politique et de transmission culturelle. Le choc violent des événements internationaux et italiens paraissait avoir précipité une désaffection qui avait des racines plus profondes (Baccetti, Caciagli, 1992).

\section{Vers la seconde république : (1992- 1994)}

Les scores électoraux de 1992 vont indi

Après le bouleversement de 1990, le résultat des législatives de 1992 traduit l'enregistrement d'un tremblement de terre déjà avéré, une issue à contre-courant d'une tendance nationale où se met en marche, de manière décisive, l'effondrement du système partisan italien. La faiblesse de la Lega Nord et les replis limités de la $\mathrm{DC}$ révèlent une situation de moindre discontinuité en Toscane vis-à-vis du reste du pays. L'électorat régional montre, en dépit de tout ce qui s'est passé, une stabilité certaine, une viscosité imperméable au changement par rapport aux crises affectant presque toutes les autres régions italiennes. C'est cette stabilité de fond, elle-même, qui préfigure les résultats de 1994 et 1996, où l'on assiste à une continuité du comportement électoral toscan, en net contraste avec les mutations radicales qui affectent le système politique italien (Baccetti, 1997). À cette persistance d'orientation électorale vient s'ajouter une habile stratégie d'alliance que le PDS a commencé à mettre en cuuve à l'occasion des premières élections municipales partielles de 1993.

Lintroduction des nouvelles règles électorales municipales, avec l'élection directe du maire et le système majoritaire, fera naitre en Toscane, entre l'été et l'automne 1993, des coalitions opposées. Ce cadre favorisera largement celles guidées par le PDS qui, en Toscane comme ailleurs, fit de ces élections partielles la première étape de son regain.

La DC et le PSI toscans sont à l'inverse frappés par la crise et la disparition de leurs partis nationaux respectifs. Les démocrateschrétiens ne semblent pas convaincus que leur parti disparaisse, et restent passifs, pendant toute l'année 1993, face aux événements nationaux : la création du Parti Populaire et du Centre Chrétien Démocrate n'y intervient que par réflexe des recompositions romaines. Ces deux partis naissent très faibles, en janvier 1994. Du PSI ne reste qu'une patrouille aguerrie de gauche qui compose le groupe des Laburisti. Sur la droite, alors que s'opère lentement la transformation du MSI en Alleanza Nazionale (AN), les tentatives de donner vie à Forza Italia rencontrent beaucoup de difficultés.

Dans la perspective des premières élections reposant sur le nouveau système majoritaire à la Chambre des Députés et au Sénat, la composition des listes progressistes ne soulève pas de difficulté, hormis quelques excès de personnalisation. À cette liste, clairement dominée par le PDS, adhéreront, outre Rifondazione Comunista, les Laburisti et d'autres groupes ex-socialistes mineurs, les Verts, les Chrétiens sociaux, et les libérodémocrates de l'Alleanza Democratica. Au centre de l'échiquier, le Pacte pour l'Italie ras- 


\section{Pôle Sud $N^{\circ} 8$}

\begin{tabular}{|c|c|c|c|c|}
\hline \multicolumn{5}{|c|}{$\begin{array}{c}\text { 3. Toscane. Elections législatives (députés) } \\
\text { 1987-1992-1994-1996. } \\
\text { Résultats des votes de liste (en \%) }\end{array}$} \\
\hline \multirow{3}{*}{$\begin{array}{l}\text { Partis } \\
\text { PDS } \\
\text { PRC }\end{array}$} & \multirow[t]{3}{*}{1987} & 1992 & 1994 & 1996 \\
\hline & & 29,7 & 33,7 & 34,7 \\
\hline & & 9,6 & 10,1 & 12,5 \\
\hline PCI & 43,3 & & & \\
\hline $\mathrm{DP}$ & 1,9 & & & \\
\hline Verdi & 2,7 & 2,7 & 2,4 & 2,0 \\
\hline Alleanza democratica & & & 1,4 & \\
\hline La Rete & & 1,3 & 1,3 & \\
\hline PSI & 13,0 & 12,8 & 2,5 & \\
\hline PSDI & 1,3 & 1,6 & & \\
\hline PRI & 3,3 & 5,0 & & \\
\hline DC & 25,8 & 22,1 & & \\
\hline PPI & & & 8,3 & \\
\hline Patto Segni & & & 5,9 & \\
\hline Popolari per Prodi & & & & 5,7 \\
\hline Lista Dini & & & & 4,3 \\
\hline CCD-CDU & & & & 4,8 \\
\hline PR/Lista Panella* & 2,0 & 1,3 & 3,8 & 1,9 \\
\hline PLI & 1,1 & 2,4 & & \\
\hline Forza Italia & & & 16,4 & 14,3 \\
\hline MSI-DN & 4,4 & 4,5 & & \\
\hline $\mathrm{AN}$ & & & 10,9 & 15,8 \\
\hline Lega Nord ** & 0,3 & 3,1 & 2,2 & 1,8 \\
\hline CPA & 0,7 & 1,6 & & \\
\hline Autres & 0,2 & 2,3 & 1,1 & 2,2 \\
\hline Votants & 93,4 & 91,1 & 91,2 & 88,4 \\
\hline Nuls & 4,2 & 5,2 & 4,8 & 5,2 \\
\hline Dont blancs & 1,9 & 2,3 & 2,3 & 2,4 \\
\hline
\end{tabular}

*En 1987 Partito Radicale; en 1992 et 1994 Lista Panella; en 1996 Lista Panella-Sgarbi

** En 1987 Liga Veneta; en 1992 Lega Lombarda

semble les nouveaux Popolari et les partisans de Mario Segni. Forza Italia, qui entendait former ses listes avec de nouvelles personnalités, socioprofessionnelles, industrielles et intellectuelles, eut des difficultés à trouver en Toscane ce type de candidat et dut souvent avoir recours à des hommes politiques de second plan de la DC et du PSI. Comme dans le Nord, Forza Italia s'allia à la Lega Nord et le CCD dans le Pôle de la Liberté. $\mathrm{AN}$, contrainte par le veto de la Lega à se présenter seule, dut recourir aux hommes du vieux MSI pour composer ses listes.

Les progressistes parviendront à obtenir une nette victoire, en conquérant les 29 sièges uninominaux de la Chambre et les 14 du Sénat, avec un taux de, respectivement, $49,6 \%$ et $49,1 \%$ des suffrages. Le premier opposant, le Pôle de la Liberté, ne parviendra qu'à $20,1 \%$ et $17,6 \%$. On peut voir (tableau 3) les résultats concernant la partie proportionnelle qui attestent de la montée du PDS et de Rifondazione Comunista, mais aussi des ex-fascistes qui doublent les scores traditionnels du MSI. Le même tableau montre la faiblesse de Forza Italia en Toscane et l'écroulement des ex-démocrateschrétiens. Les élections de 1994, semblent ainsi mettre fin aux tourments qui avaient affecté la gauche depuis 1989.

Naturellement, les progressistes ont de meilleurs résultats dans les zones où l'enracinement électoral et organisationnel du PCI était le plus profond. Dans celles-ci, dont les situations socio-économiques étaient par ailleurs fort diverses, l'écart entre les pourcentages des progressistes (autour de $60 \%$ ) et ceux du Pôle (environ $20 \%$ ) est énorme. Selon la tradition, les premiers ont leur plus mauvais score dans la province de Lucca, où la tradition catholique favorise le PPI. Ils ont également des résultats négatifs dans les circonscriptions de Carrare, Grosseto et Versilia où ils ne doivent leurs conquêtes en sièges qu'à la division de la droite et à la présence du centre ex-démocrate-chrétien. C'est sur ces zones marginales que reposeront, deux ans après, les espoirs de revanche du Pôle.

Ces chiffres, supérieurs pour les progressistes à la somme des votes obtenus en 1992 par le Pds et le Prc, traduisent, d'une part, le retour de beaucoup d'électeurs 


\section{Dynamiques électorales et forces politiques en Toscane}

ex-communistes au vote, et au vote à gauche, et, d'autre part, l'orientation à gauche d'une fraction de l'électorat modéré.

\section{Une nouvelle carte du pouvoir local}

1995 marque une autre étape dans la stratégie d'alliance du PDS, qui redessine la carte du pouvoir local toscan. Le facteur décisif, dans la stratégie des élections régionales et locales de cette année-là, est l'alliance avec le Parti Populaire, que nous considérons comme un véritable tournant historique. À cette occasion, une partie de ses adversaires

4. Toscane. Composition des coalitions partisanes des conseils municipaux après les élections de 1990. (Données absolues et \%)

\begin{tabular}{|c|c|c|}
\hline PARTIS ET COALITIONS & $N$ & $\%$ \\
\hline Monocolore PCI & 92 & 32,0 \\
\hline PCI + PSI & 60 & 20,9 \\
\hline $\mathrm{PCI}+\mathrm{CPA}$ & 1 & 0,3 \\
\hline PCI + PSDI & 1 & 0,3 \\
\hline PCI + Verdi & 5 & 1,7 \\
\hline PCI + PSI + PSDI & 13 & 4,5 \\
\hline $\mathrm{PCI}+\mathrm{PSI}+$ Verdi & 2 & 0,7 \\
\hline PCI + PSI + PRI & 9 & 3,1 \\
\hline PCI + PI + PRI + PSDI & 11 & 3,8 \\
\hline $\mathrm{PCI}+\mathrm{PSI}+\mathrm{PSDI}+\mathrm{Verdi}$ & 2 & 0,7 \\
\hline $\mathrm{PCI}+\mathrm{DC}$ & 3 & 1,1 \\
\hline $\mathrm{PCI}+\mathrm{DC}+\mathrm{Verdi}$ & 1 & 0,3 \\
\hline $\mathrm{PCI}+\mathrm{DC}+\mathrm{CPA}$ & 1 & 0,3 \\
\hline \multicolumn{3}{|l|}{ Total conseils municipaux } \\
\hline avec PCI & 201 & 70 \\
\hline $\mathrm{DC}$ & 32 & 11,1 \\
\hline $\mathrm{DC}+\mathrm{PSI}$ & 26 & 9,0 \\
\hline $\mathrm{DC}+\mathrm{PSDI}$ & 3 & 1,1 \\
\hline $\mathrm{DC}+\mathrm{CPA}$ & 2 & 0,7 \\
\hline $\mathrm{DC}+$ Verdi & 2 & 0,7 \\
\hline $\mathrm{DC}+\mathrm{PSI}+\mathrm{PRI}$ & 4 & 1,3 \\
\hline $\mathrm{DC}+\mathrm{PSI}+\mathrm{PSDI}$ & 9 & 3,1 \\
\hline $\mathrm{DC}+\mathrm{PSDI}+\mathrm{PLI}$ & 1 & 0,3 \\
\hline $\mathrm{DC}+\mathrm{PSI}+\mathrm{PSDI}+\mathrm{PRI}$ & 4 & 1,3 \\
\hline $\mathrm{DC}+\mathrm{PLI}+\mathrm{PSDI}+\mathrm{PRI}$ & 2 & 0,7 \\
\hline Pentapartito & 1 & 0,3 \\
\hline \multicolumn{3}{|l|}{ Total conseils municipaux } \\
\hline sans PCI & 86 & 30,0 \\
\hline Total de conseils municipaux & 287 & 100 \\
\hline
\end{tabular}

traditionnels se rapproche des héritiers du $\mathrm{PCI}$, favorisant une concentration politique largement supérieure à toutes celles passées.

Le tableau 4 rappelle la composition des coalitions des partis de gouvernement municipal toscan après les élections de 1990 . Il en découle que le PCI était présent dans 201 communes sur 287 , soit environ $70 \%$, et gouvernait seul dans 92 (le tiers des municipalités toscanes). On notera également que le PSI était son partenaire majeur, où il lui était allié dans un autre tiers des communes. Le PSI était allié à la DC dans un nombre limité de communes.

Le tableau 5 montre de quelle manière la carte du pouvoir local change radicalement après les élections de 1995. Le PDS est désormais présent dans l'exécutif de 261 communes sur 287 , soit $91,3 \%$. La grande majorité de celles-ci $(178$, soit $62 \%)$ est gouvernée par des exécutifs de centre gauche, où le principal partenaire du PDS est le PPI. L'alliance avec l'héritier principal de la DC, souvent représenté par des ex-démocrates-chrétiens de gauche, a même permis l'extension du pouvoir du PDS toscan dans les zones et villes de tradition catholique (la première d'entre elles : Lucca) qui étaient restées fermées au PCI. Il est également intéressant de noter que dans 84 communes (29,3\%), le PDS s'allie avec Rifondazione Comunista et d'autres partis mineurs de gauche, en excluant le PPI et les petits partis du centre. Ceci indique d'un côté que la gauche est en mesure de gouverner seule un tiers des municipalités, mais montre aussi la condition minoritaire qui est faite à Rifondazione, et sa marginalisation malgré l'influence électorale et organisationnelle dont elle jouit en Toscane. Le tableau témoigne, sil était encore besoin, de la faiblesse du centredroit, qui est encore plus évidente dans le paysage du pouvoir local où le manque de 


\section{Pôle Sud $N^{\circ} 8$}

5. Toscane. Composition des coalitions politiques des conseils municipaux après les élections de 1995. (Données absolues et \%)

\begin{tabular}{lrr}
\hline PARTIS ET COALITIONS & $\mathrm{N}$ & $\%$ \\
Progressisti & 34 & 11,9 \\
PDS & 37 & 12,9 \\
PDS + PRC & 13 & 4,5 \\
Conseils de gauche & 84 & 29,3 \\
Centre gauche & 159 & 55,4 \\
PDS + PSI & 4 & 1,4 \\
PDS + PRI & 3 & 1,1 \\
PDS + PSI + PRI & 9 & 3,1 \\
PDS + PRI + Verdi & 1 & 0,3 \\
PDS + PPI + Verdi & 1 & 0,3 \\
PDS + PPI + Lega Nord & 1 & 0,3 \\
Conseils de centre gauche (total) & 178 & 62,0 \\
Conseils de gauche et & & \\
centre gauche avec PDS & 262 & 91,3 \\
DC & 5 & 1,7 \\
PPI & 4 & 1,4 \\
Centro & 4 & 1,4 \\
DC + CPA & 1 & 0,3 \\
Conseils du centre (total) & 14 & 4,5 \\
Centre droit & 8 & 2,7 \\
PPI + AN & 2 & 0,7 \\
PPI + Forza Italia & 1 & 0,3 \\
Conseils de centre-droit (total) & 11 & 3,8 \\
Conseils sans PDS & 25 & 8,7 \\
Nombre de conseils & 287 & 100 \\
\hline
\end{tabular}

candidats pertinents, soit par disponibilité personnelle, soit par expérience politicoadministrative, a pénalisé les positions conservatrices plus que dans les élections parlementaires.

En 1995, le PDS a également fait un pas en avant dans les élections régionales, comme on peut le voir dans le tableau 2. En fait, la somme des votes PDS et Rifondazione dépasse largement la moitié ( $52 \%$ ), un score jamais obtenu dans le passé par le PCI. Ceci peut indiquer la capacité du PDS à étendre son influence vers des fractions de l'électorat de tradition non-communiste. Dans la coalition nécessaire pour élire le président de la région et conquérir ainsi la prime majoritaire, le PDS a réussi à agréger sous le nom de Toscana Democratica, un ensemble composé d'ex-socialistes, d'ex-libéraux, d'ex-républicains, jusqu'à la Lega Nord et, par-dessus tout, les ex-démocrates-chrétiens du PPI et de Segni. Toscana Democratica obtient $50,1 \%$, alors que la coalition de centre-droit formée par Forza Italia, AN et le CCD n'obtient que 36,1 \%. Rifondazione Comunista, restée en dehors de la coalition de centre gauche, et donc de la compétition régionale, obtient toutefois le score relativement élevé de $12,4 \%$ et constitue l'opposition de gauche au Conseil régional.

\section{Le grand succès de l'olivier}

Les succès des coalitions de centre gauche lors des élections municipales et régionales de 1995 constituent, en Toscane plus qu'ailleurs, un terrain fertile pour l'affirmation de la nouvelle coalition formée autour de la candidature de Romano Prodi à la présidence du Conseil : l'Olivier. Le PDS a été l'axe moteur de la formation des listes et de la campagne électorale. Une négociation habile avec les forces centristes de l'Olivier a garanti à la fois au groupe du Président du Conseil en exercice, Lamberto Dini, la capacité (comme on l'a vu dans la quote-part proportionnelle) d'attirer un vote modéré, et au PPI la visibilité nécessaire pour attirer le vote catholique (Baccetti, 1997). Mais, précisément pour cela, le PDS a été contraint de céder beaucoup de circonscriptions sûres aux alliés plus forts (le PPI ou Rifondazione) ou plus utiles parce que modérés. Les comités Prodi, nés un an auparavant en Toscane, protesteront en revanche contre le fait de n'avoir pas pu obtenir une seule circonscription sûre. La négociation pour l'attribution des candidatures au sein d'une coali- 


\section{Dynamiques électorales et forces politiques en Toscane}

tion aussi complexe (environ 10 groupes) était de fait difficile et laborieuse.

Les négociations pour désigner les candidatures uninominales ont été plus faciles dans le camp opposé. La compétition interne était inévitablement plus réduite, parce que peu de collèges électoraux promettaient une issue favorable au centre-droit, même si la nouveauté de l'alliance entre Forza Italia et AN, par rapport à 1994, semblait ouvrir quelque opportunité de conquête locales. En Toscane comme ailleurs, le Pôle comptait récupérer une partie de l'électorat démocrate-chrétien qui avait voté PPI auparavant.

Mais, une fois ouvertes les urnes, on a pu voir que l'Olivier avait été nettement plus attractif pour les électeurs du centre, y compris pour une grande partie des ex-démocrates-chrétiens. Dans la compétition, le centre gauche est parvenu à conquérir cet électorat, probablement encore d'inspiration catholique, grâce à la tradition antifasciste de la région, hostile au parti post-fasciste et à ses alliés. Le centre-gauche a ainsi obtenu tous les sièges au Sénat et à la Chambre, avec une seule exception, à Grosseto, où une personnalité nationalement connue, l'ex-magistrat Tiziana Parenti, a réussi à s'affirmer.

La coalition de l'Olivier a atteint $52 \%$ dans l'ensemble des 25 collèges uninominaux, démontrant ainsi sa capacité à attirer à la fois l'électorat de Rifondazione et celui des modérés. Dans les 4 autres collèges de la Chambre, l'Olivier appuyait le candidat de Rifondazione, en vertu du pacte de désistement décidé à l'échelle nationale : dans ces collèges, les candidats de gauche ont obtenu $8 \%$ qui, ajoutés aux $52 \%$ de centre gauche, confirmaient l'orientation progressiste de la région.
Les analyses approfondies ont montré que le report des voix sur les candidats " éloignés dans la coalition " a été plus massif pour Rifondazione et le PDS que pour les modérés, selon une fidélité aux pactes typiques de la subculture rouge. Ainsi l'élément traditionnel de discipline électorale communiste et ex-communiste - un trait culturel régional important - est venu s'ajouter à une utilisation stratégique du vote, propre à la compétition uninominale. L'ampleur de la convergence d'électeurs d'origines diverses a été en outre facilitée par un climat substantiellement unitaire dans la campagne que les différentes composantes de l'Olivier ont su construire, et par l'effort de Rifondazione pour ne pas accentuer (comme c'était le cas en 1994) les éléments de clivages programmatiques entre forces politiques appartenant à la coalition (Floridia, 1996, p. 39).

La distribution en pourcentage des votes par liste en 1996 (tableau 3) montre combien la somme des votes PDS et PRC $(47,2 \%)$ dépasse nettement la part obtenue par le PCI en 1987 (43,3\%). Ceci indique au moins une récupération des positions antérieures, même si l'on est encore loin de l'expansion promise par les fondateurs du PDS. À ce propos, on peut observer que si le PDS progresse vis-à-vis des élections locales précédentes, et s'il est largement confirmé comme premier parti toscan, il subit une chute notable de 6 points par rapport au triomphe des élections régionales de l'année antérieure. Ceci peut signifier que la fonction de parti de gouvernement à la région laisse encore une marge de progression élevée auprès de l'électorat.

On doit signaler surtout la constante progression de Rifondazione dans tous les types 


\section{Pôle Sud $N^{\circ} 8$}

de consultation, comme témoignage de la fidélité aux vieux idéaux communistes, qui rejoint dans la région le malaise des jeunes et des exclus.

Sur le versant centre-droit, la progression de l'AN est constante sur trois élections différentes, et elle l'a même conduit à dépasser Forza Italia en 1996, et à se présenter comme l'unique alternative à la gauche. Forza Italia a subi en fait, lors des dernières élections, de graves pertes de position par rapport aux régionales, en ne parvenant pas à conquérir une réelle position de protagoniste.

Nonobstant le succès de l'Olivier auquel, comme on l'a dit, l'électorat ex-DC a certainement contribué, les popolari ont obtenu un modeste $5,7 \%$, en baisse par rapport aux deux élections précédentes. En dépit de la richesse de sa mémoire et de son activisme associatif, la minorité catholique n'a plus les moyens électoraux de ses ambitions.

\section{Les forces politiques : un panorama uniforme}

Si les dynamiques électorales montrent, après une décennie de bouleversement de tout le système italien, la capacité des héritiers du PCI à confirmer l'influence du vieux parti sur la région, un recensement des organisations politiques révèle une accentuation de la prédominance de la gauche en Toscane.

La DC et le PSI avaient eu, comme on l'a vu, un nombre très modeste d'inscrits, tant par rapport au PCI toscan que vis-à-vis de leur parti à l'échelle nationale. Une fois ces derniers disparus, les ex-socialistes constituent désormais de petits cercles, modestes en quantité et en conflits internes permanents. Parmi les socialistes, seuls les Laburisti, grâce au dynamisme de leur leader, le Florentin Valdo Spini, réussissent à avoir une présence visible, en termes de maire-adjoints et de conseillers municipaux.

La diaspora démocrate-chrétienne a engendré, comme on le sait, outre le PPI, le CCD et le CDU. Quelques personnalités de ces partis parviennent à jouir d'une image régionale et nationale, à laquelle ils cherchent à faire correspondre un poids politique à l'intérieur des coalitions respectives. En revanche, ils n'ont que quelques milliers d'adhérents, présents, selon le cas, dans une ville ou dans une province seulement. Le destin des ex-DC toscans semble ainsi lié à leur capacité à avancer à l'ombre de leur référent majeur : une perspective qui paraît plus heureuse pour le PPI, lié au puissant PDS, quau $C C D$ et au CDU, alliés à une faible Forza Italia en conflit continuel avec les postfascistes d'AN. Forza Italia, comme on l'a vu, n'est pas parvenue, même quand $S$. Berlusconi l'avait décidé et tenté de le faire, à assumer le statut d'un véritable parti. C'est encore moins le cas en Toscane qu'ailleurs : il existe bien un secrétariat régional, quelques congrès provinciaux sont bien organisés et les élus à tous les niveaux peuvent bien chercher à instaurer des relations organiques avec leur base électorale. Mais l'impression qu'ils donnent est celle d'officiers en manœuvre sans soldats. Outre les problèmes organisationnels, Forza Italia ne semble pas réussir à trouver en Toscane une consistance et une base sociale durable de référence. Seules des erreurs à gauche lui ont permis d'obtenir quelques niveaux majoritaires.

L'organisation et le recrutement d'AN sont sûrement plus consistants. En Toscane, plus qu'ailleurs, elle s'appuie quasi- 


\section{Dynamiques électorales et forces politiques en Toscane}

exclusivement sur l'héritage du MSI. Le fait que l'AN ne fournisse aucune donnée officielle sur sa base régionale ou provinciale, fait qu'il est difficile d'établir que le parti soit dans une phase d'extension parallèle à sa progression électorale. Cependant, il est clair que les ex-dirigeants du MSI, qui sont aujourd'hui députés, sénateurs ou conseillers régionaux ou provinciaux se sentent membres à part entière de la vie politique régionale, après être restés pendant un demi-siècle dans un ghetto.

Tout compte fait, les seuls partis qui, en termes d'inscrits, de structures de base et d'organismes périphériques peuvent être considérés comme tels sont, en Toscane, le PDS et Rifondazione comunista.

Le PDS a démantelé, même dans la région rouge, l'imposant appareil bureaucratique du PCI, et Rifondazione n'a pas constitué une bureaucratie digne de ce nom. Mais les deux ont en Toscane un nombre d'inscrits et de structures territoriales (sections, unions communales et fédérations provinciales) qui ont indiscutablement une importance exceptionnelle dans la transition italienne, quand tous les partis du passé sont entrés irrémédiablement en crise ou ont disparu. Certes, les chiffres des inscrits sont loin de ceux du vieux PCI, mais les adhérents au PDS sont encore environ 95000 ( $15 \%$ du total national) et ceux de Rifondazione quasi 20000 (également $15 \%$ du total italien, et le plus élevé par région).

On ne peut oublier, en outre, qu'autour des deux partis, continue d'opérer un réseau d'institutions qui exprime une tradition vivace : les associations de loisir, les maisons du peuple, les groupes de volontaires, les fêtes populaires. Ce sont ces institutions qui parviennent encore à mobiliser et à organiser une part notable des citoyens toscans, au nom d'une sociabilité vieille d'un siècle. À cette tradition appartiennent aussi les coopératives, encore nombreuses, riches et puissantes dans la région : si l'encadrement managérial y domine aujourd'hui, leur fonction de support aux deux partis est encore irremplaçable.

Les syndicats continuent à atteindre des taux élevés en Toscane, malgré une désaffection qui, à travers la crise du système représentatif actuelle, les a également touchés. Le plus puissant, en nombre d'inscrits, est naturellement la CGIL : comme avant, il continue à recruter les $2 / 3$ des travailleurs syndiqués en Toscane (dans l'ensemble, c'est-à-dire les retraités compris, les adhérents à la CGIL ont été en moyenne 450 000 dans les années 1980).

Dans tous ces organismes collatéraux, la présence du PDS et celle de Rifondazione se superposent et se coagulent, malgré des tensions et des polémiques parfois féroces. Le militantisme commun d'un temps occasionne de durs conflits mais aussi des combats communs, en particulier lors des compétitions électorales.

\section{Les transformations de la culture}

\section{rouge}

La Toscane apparaît ainsi fidèle à son passé, tant dans ses orientations électorales majeures que dans les modes d'organisation de sa vie politique. Le regain de la gauche, en termes d'influence aussi bien qu'en termes de conscience de ses propres forces et capacités de gouvernement, a été probablement 


\section{Pôle Sud $N^{\circ} 8$}

possible grâce à la bipolarisation engendrée par le nouveau système électoral et par la restructuration du système partisan à partir de 1993. L'émergence d'un parti post-fasciste et d'un " parti-entreprise " ${ }^{3}$ comme seuls adversaires de la gauche a facilité la recomposition d'un électorat désorienté et prompt à la dispersion, comme l'électorat communiste des années 1990-1992. Le protagonisme de l'AN au plan national a réveillé le profond sentiment antifasciste des citoyens toscans, qui semblent faire peu de cas de l'évolution de G. Fini et des siens; les déclamations libérales et individualistes de $S$. Berlusconi et son initiative improvisée paraissent éloignées de la mentalité désenchantée de la majeure partie des Toscans.

Tout ceci signifie que le retour de la gauche peut être attribué, en cette période de transition, à une réaction négative plus qu'à une orientation constructive. Il n'est donc pas dit que les succès électoraux du PDS et de Rifondazione aient la solidité et puissent avoir la continuité multi-décennale propres au PCI. C'est l'inconsistance d'une alternative crédible de centre-droit qui favorise l'option de gauche des électeurs toscans. Mais ceci ne doit pas faire croire que la substance de la culture rouge est restée inchangée. Les deux partis de gauche ont une organisation plus faible que celle du PCI, et un nombre d'inscrits largement inférieur. Pour ne rien dire des autres, presque inexistants, le PDS et Rifondazione ont une présence peu visible et peu influente dans la société; leurs liens avec les électeurs sont donc devenus moins solides; les associations collatérales ont incontestablement une plus grande autonomie à leur égard. Bien des valeurs et des mythes de l'ancienne culture politique locale ont été bouleversés par l'histoire ou étouffés par les profondes mutations économiques. Le bien-être a affaibli la solidarité pendant qu'il faisait croître l'individualisme.

D'un point de vue spécifiquement politique, malgré une incontestable crise de la participation, la société Toscane demeure cependant fortement politisée et riche en structures associatives, qui lui permettent d'appartenir, comme dans le passé, au monde d'une culture civique qu'on lui attribue comme d'autres régions italiennes (Putnam, 1993). Cette culture civique et la solidité des rapports avec les institutions locales, l'entrelacement d'intérêts matériels et de biens symboliques paraissent avoir résisté à la crise idéologique des années 1990 , et avoir ainsi tenu la région à l'écart des tensions qu'on peut ressentir dans d'autres parties du pays. 


\section{Dynamiques électorales et forces politiques en Toscane}

\section{Notes}

1. Le malaise avait déjà pointé à travers le succès de la liste Chasse, Pêche et Environnement (CPA) lors des élections régionales et locales.

2. En vertu de la nouvelle loi électorale pour les régions ( $\left.{ }^{\circ} 43 / 1995\right)$, si $4 / 5$ des sièges continuent à être attribués à la proportionnelle sur une base provinciale, $1 / 5$ (en Toscane : 10 sièges) est attribué en totalité à la coalition régionale qui conquiert le plus grand nombre de suffrages. Si la coalition obtient déjà la majorité absolue des sièges à la proportionnelle, la prime majoritaire redescend à $10 \%$ des sièges.

3. Le cas le plus symbolique fut celui de l'ex-président du Conseil, Lamberto Dini, qui a obtenu le soutien total de l'électorat de Rifondazione dans une circonscription de la ville de Florence, malgré la défiance exprimée contre lui par ce parti.

\section{Références}

Baccetti C., "L'ultima svolta. Il XIX congresso del PCI in Toscana ", Polis, $\mathrm{n}^{\circ} 3,1991$.

Baccetti C., "La Toscana : le nuove vesti di un'antica egemonia ", in Gangemi G., Riccamboni G. (dir.), Le elezioni della transizione, Torino, Utet, 1997.

Baccetti C., Caciagli M., " Dal divorzio alla caccia. Il declino della subcultura rossa ", in Caciagli M., Uleri P. V. (dir.), Democrazie e referendum, Roma-Bari, Laterza, 1994.

Baccetti C., Caciagli M., " Dopo il PCI e dopo l'URSS. Una subcultura rossa rivisitata " Polis, n 3, 1992.

Caciagli M., "Il declino della subcultura rossa ", in Feltrin P., Politi A. (dir.), Elezioni regionali del 90 . Un punto di svolta, Venezia, Le Fondazione Corazzin, 1990.

Caciagli M., "La destinée de la subculture rouge dans le Centre Nord de l'Italie " Politix, n 30, 1995.

Floridia A., "La metamorfosi di una regione rossa : stabilità ed evoluzione nel voto del 21 aprile 1996 in Toscana ", Quaderni dell'Osservatorio elettorale, nº 36, 1996.

Putnam R., Making democracy Work, Princeton, Princeton University Press, 1993. 